\title{
Qualidade da dieta de indivíduos expostos e não expostos a um programa de reeducação alimentar
}

\author{
Diet quality of individuals exposed or not to \\ a nutrition education program
}

Flavia FELIPPE 1

Liziana BALESTRIN²

Flávia Moraes SILVA ${ }^{3}$

Aline Petter SCHNEIDER ${ }^{4}$

RE S U M O

\section{Objetivo}

O presente estudo teve como objetivo comparar a qualidade da dieta de indivíduos expostos e não expostos a um programa de reeducação alimentar.

\section{Métodos}

Foi realizado estudo transversal, envolvendo 100 indivíduos, com idade média de 40,7, DP=12,4 anos e índice de massa corporal médio de $27,4, \mathrm{DP}=4,7 \mathrm{~kg} / \mathrm{m}^{2}$. Foram avaliados dois grupos: um grupo com 54 indivíduos expostos a um programa de reeducação alimentar por 18 meses (grupo E) e um grupo composto por 46 indivíduos sem orientação nutricional prévia nos últimos 6 meses (grupo-controle). Ambos os grupos foram submetidos a avaliação antropométrica (peso, estatura, índice de massa corporal e circunferência da cintura) e do consumo alimentar (dois recordatórios alimentares de 24 horas). A análise dietética foi realizada no software Avanutri ${ }^{\circledR}$, e a qualidade da dieta foi estimada pelo Índice de Alimentação Saudável adaptado para a população brasileira.

\section{Resultados}

A pontuação no Índice de Alimentação Saudável, adaptado para a população brasileira, foi maior no grupo $E$ do que no grupo-controle ( $M=97,0, D P=6,2$ vs $M=84,2, D P=15,2 ; p<0,001)$. Em relação às categorias de classificação desse índice, os grupos diferiram quanto ao percentual de indivíduos com dieta de boa qualidade (29,6\% dos indivíduos do grupo E vs $17,4 \%$ dos indivíduos do grupo-controle) e de má qualidade (0\% no

\footnotetext{
1 Assistente Social. Porto Alegre, RS, Brasil.

2 Nutricionistas. Porto Alegre, RS, Brasil.

${ }^{3}$ Hospital das Clínicas de Porto Alegre, Serviço de Endocrinologia. R. Ramiro Barcelos, 2400, Santa Cecília, 90035-003, Porto

Alegre, RS, Brasil. Correspondência para/Correspondence to: F.M. SILVA. E-mail: <flavia.moraes.silva@hotmail.com>.

${ }^{4}$ Universidade Federal do Rio Grande do Sul, Faculdade de Medicina, Curso de Nutrição, Departamento de Medicina Social. Porto Alegre, RS, Brasil.
} 
grupo E vs 10,9\% no grupo-controle). Os indivíduos expostos ao programa de reeducação alimentar apresentaram maior pontuação para vegetais, frutas, laticínios, colesterol e variedade, assim como menor pontuação para óleos e açúcares, em comparação ao grupo-controle.

\section{Conclusão}

Indivíduos expostos ao programa de reeducação alimentar apresentaram dieta com melhor qualidade do que indivíduos sem acompanhamento nutricional prévio. Tais achados reforçam a importância da educação nutricional na promoção da alimentação saudável.

Termos de indexação: Avaliação nutricional. Comportamento alimentar. Consumo alimentar. Dieta. Educação alimentar e nutricional.

\section{A B S T R A C T}

\section{Objective}

The present study compared the diet quality of individuals exposed or not to a nutrition education program.

\section{Methods}

This cross-sectional study compared the diets of 54 individuals exposed to a nutrition education program for 18 months (varied between 2 and 169 months) (group E) to those of 46 individuals who did not receive nutritional counseling in the last six months (group NE). All participants underwent anthropometric (weight, height, body mass index, waist circumference) and dietary assessments (two 24-hour recalls). Nutrient intakes were calculated by the Avanutri software ${ }^{\circledR}$ and diet quality was estimated by the Healthy Eating Index adapted for the Brazilian population (IASad).

\section{Results}

A total of 100 individuals with a mean age of 40.7, SD=12.4 years and mean body mass index of 27.4, $S D=4.7 \mathrm{~kg} / \mathrm{m}^{2}$ were studied. The two groups did not differ significantly in terms of age and BMI. Group $E$ had a higher IASad score than group NE (97.0, SD=6.2 vs 84.2, SD=15.2, p<0.001). Regarding IASad classification categories, the percentages of individuals consuming a good-quality diet in the two groups differed significantly (29.6\% in group E vs $17.4 \%$ in group NE) as did the percentages of those consuming a poor-quality diet $(0 \%$ in group $E$ versus $10.9 \%$ in group $N E, p=0.014)$. Group $E$ scored higher than group NE for vegetables, fruits, dairy, cholesterol and diet variety and lower than group NE for oils and sugars.

\section{Conclusion}

Individuals exposed to a nutrition education program had a better-quality diet than individuals not exposed to nutritional counseling. These findings reinforce the importance of nutrition education for the promotion of healthy food habits.

Indexing terms: Nutrition assessment. Feeding behavior. Food consumption. Diet. Food and nutrition education.

\section{N T R O D U ÇÃ O}

A alimentação nutricionalmente adequada pode atuar tanto na prevenção como no tratamento de doenças. Nesse sentido, torna-se cada vez mais evidente a importância da avaliação dos hábitos alimentares da população. A avaliação da qualidade da dieta de indivíduos e/ou grupos populacionais é essencial ao conhecimento de padrões alimentares e ao estabelecimento das relações de causalidade entre dieta e doenças.

Os índices dietéticos têm sido desenvolvidos para fornecer uma medida resumo das prin- cipais características da dieta, facilitando a avaliação da qualidade da dieta em populações ou grupos de indivíduos ${ }^{1}$. Esses índices consideram diferentes parâmetros, tais como grupos alimentares, nutrientes específicos, variedade e/ou diversidade. Dentre os instrumentos para avaliação da qualidade da dieta descritos na literatura, podem-se citar o Índice de Nutrientes (IN), o Escore de Variedade da Dieta (EVD), o Escore de Diversidade da Dieta (EDD), o Índice de Qualidade da Dieta (IQD) e o Índice de Alimentação Saudável (IAS)².

Considerar nutrientes, alimentos e grupos de alimentos em conjunto parece ser uma ótima 
abordagem para análises epidemiológicas da dieta, a fim de se obter o máximo de informações acerca desta ${ }^{3}$. Nesse sentido, os índices dietéticos baseados em alimentos e nutrientes podem ser mais promissores, pois retêm a complexidade da dieta e permitem uma avaliação indireta de nutrientes, sem reduzir a avaliação a um único componente isolado'.

Atendendo a essas características, destaca-se o Índice de Alimentação Saudável norte-americano (IAS), desenvolvido em $1995^{4}$ e atualizado a cada cinco anos, o qual considera em sua última versão $(2005)^{5}$ os seguintes componentes: cereais totais, cereais integrais, vegetais totais, vegetais verdes escuros e legumes alaranjados, frutas totais, frutas in natura, leite e derivados, óleos, carnes, leguminosas, gordura saturada, sódio e calorias provenientes de gordura sólida, álcool e açúcares. A soma dos 12 componentes totaliza um valor máximo de 100 pontos e reflete a aderência às recomendações nutricionais para a população americana ${ }^{6}$.

Em 2008, Mota et al. ${ }^{7}$ apresentaram uma adaptação do IAS norte-americano (versão original de 1995) à população brasileira, considerando as recomendações nutricionais do país para uma alimentação saudável ${ }^{8}$, denominando o instrumento de Índice de Alimentação Saudável Adaptado (IASad). O IASad diferencia-se do IAS americano original, pois considera o número de porções dos grupos alimentares preconizados na Pirâmide Alimentar Brasileira Adaptada ${ }^{9}$, inclui o grupo das leguminosas, dos doces e açúcares e dos óleos e gorduras, exclui o item sódio da pontuação, além de estabelecer uma pontuação intermediária para o item variedade 7 .

Diversos estudos nacionais ${ }^{7,10-12}$ e internacionais ${ }^{13,14}$ avaliaram a qualidade da dieta de diferentes grupos populacionais, utilizando índices dietéticos variados. Entretanto, não foram encontrados na literatura estudos que tenham comparado a qualidade da dieta de indivíduos submetidos ou não a um programa de reeducação alimentar através de índices dietéticos. Sabe-se que a reeducação alimentar pode ser considerada uma medida para melhorar a qualidade da dieta e de vida de indivíduos e/ou populações, uma vez que fornece apoio e orientação para mudanças nos hábitos e estilo de vida ${ }^{15,16}$. A intervenção em grupos, por auxiliar no processo de identificação das pessoas que sofrem do mesmo problema e possibilitar a quebra da tradicional relação vertical que existe entre o profissional da saúde e o sujeito da sua ação, é apontada como uma estratégia facilitadora no processo de reeducação alimen$\operatorname{tar}^{17}$. Nesse sentido, o objetivo do presente estudo foi comparar a qualidade da dieta de indivíduos expostos a um programa de reeducação alimentar à dieta de indivíduos sem orientação nutricional prévia.

\section{M É T O D O S}

O presente estudo transversal foi conduzido, de um lado, em indivíduos expostos a um programa de reeducação alimentar, em uma clínica privada de Porto Alegre, especializada no atendimento multidisciplinar do excesso de peso (grupo E), de outro lado, em funcionários da prefeitura de Porto Alegre (grupo-controle). Foram incluídos no estudo homens e mulheres com idade entre 20 e 65 anos, tendo sido a amostra composta, por conveniência, entre os meses de maio e agosto de 2009. Não foram incluídas gestantes no estudo.

O grupo E foi formado por clientes de uma clínica de reeducação alimentar, os quais realizavam acompanhamento semanal em grupos de apoio voltados para mudanças de hábitos alimentares e melhoria do estilo de vida. As reuniões eram coordenadas por uma equipe multiprofissional (nutricionista, psicólogo e assistente social), que trabalhava a modificação de pensamento, ação e sentimento relacionados ao comportamento alimentar. Tais reuniões apresentavam duração média de 60 minutos e envolviam um grupo de no máximo 15 clientes. O tempo de acompanhamento na clínica variou entre os clientes que participaram do estudo, sendo a mediana de acompanhamento no programa de reeducação 
alimentar igual a 18 meses (variação: 2169 meses).

Fizeram parte do grupo-controle trabalhadores da Prefeitura de Porto Alegre, sem orientação nutricional prévia nos 6 meses anteriores à realização do estudo. O recrutamento dos participantes desse grupo foi feito a partir de cartazes com convites para avaliação nutricional e do consumo alimentar.

Todos os participantes que aceitaram participar do estudo assinaram o termo de consentimento após serem esclarecidos acerca de sua natureza. O Comitê de Ética em Pesquisa do Centro Universitário Metodista (IPA) aprovou a realização do protocolo de pesquisa (protocolo $n^{\circ} 62 /$ 2009).

Os participantes foram submetidos à avaliação nutricional e responderam a um questionário padronizado acerca de suas características clínicas e sociodemográficas.

Quanto ao hábito de fumar, foi considerado fumante o participante tabagista atual, com história de tabagismo há pelo menos 12 meses, bem como aquele que parara de fumar há menos de 6 meses da avaliação. Os demais foram considerados ex-fumantes ou não fumantes. Os participantes foram questionados quanto ao consumo de álcool e a informação obtida foi categorizada como "consome" ou "não consome". Foram questionados ainda acerca do grau de escolaridade e da prática de atividade física, sendo considerados sedentários aqueles que praticassem menos de 150 minutos de atividade física/semana ${ }^{18}$.

As medidas antropométricas utilizadas para a avaliação do estado nutricional foram o peso (com roupas leves e sem sapatos), a estatura e a circunferência da cintura (medida no ponto médio entre a última costela e a crista ilíaca) ${ }^{19}$. Para tais medidas, foram utilizadas balança antropométrica e fita métrica de fibra de vidro flexível. A partir dos dados de peso e estatura, foi calculado o Índice de Massa Corporal (IMC). Os participantes do estudo foram classificados quanto ao estado nutricional a partir do IMC, considerando-se os pontos de corte propostos pela World Health organization: eutrofia quando IMC entre 18,5 e $24,9 \mathrm{~kg} / \mathrm{m}^{2}$, sobrepeso quando IMC entre $25 \mathrm{e}$ $29,9 \mathrm{~kg} / \mathrm{m}^{2}$ e obesidade quando IMC $>30 \mathrm{~kg} / \mathrm{m}^{2} 19$.

A avaliação do consumo alimentar foi realizada através da aplicação de dois Recordatórios alimentares de 24h (R24h), com intervalo de uma semana. Os dados dietéticos obtidos em medidas caseiras foram convertidos para grama e mililitro, a fim de possibilitar a análise nutricional detalhada do consumo alimentar. A análise da composição nutricional da dieta usual de cada participante, considerando-se a média dos dois dias de R24h, foi feita no Programa AvaNutri 4.0 Revolution ${ }^{20}$.

A avaliação da qualidade da dieta foi realizada através do IAS adaptado para a população brasileira ${ }^{7}$. Para isso os alimentos consumidos foram convertidos em porções pelo valor energético, de acordo com o grupo da Pirâmide Alimentar ao qual pertenciam (Tabela 1). O número de porções recomendado para cada indivíduo foi ajustado ao valor calórico total, tendo como referência as porções recomendadas pelo Guia Alimentar para a População Brasileira ${ }^{8}$. As preparações culinárias elaboradas com mais de um grupo alimentar foram desmembradas nos seus ingredientes, e esses, classificados nos respectivos grupos correspondentes. A pontuação dos componentes do IASad variou de 0 a 10 (Tabela 1), sendo os valores intermediários calculados proporcionalmente. Dessa forma, quando o consumo dos grupos alimentares fosse igual ou superior ao recomendado, eram atribuídos 10 pontos; e, quando inferior, os pontos eram calculados por razão e proporção. Para os nutrientes (gordura total, saturada e colesterol) e para o item variedade, a pontuação também foi estabelecida por proporção entre a quantidade consumida por participante e a quantidade preconizada, conforme proposto por Mota et al. ${ }^{7}$. Tendo em vista que no presente estudo foram utilizados dois R24h, a pontuação para o item variedade foi adaptada, sendo utilizado o ponto de corte de 12 itens $(\geq 12$ 
itens) para pontuação máxima, e de 6 itens ( $<6$ itens) para a pontuação mínima. Os alimentos foram considerados como uma variedade sempre que fornecessem pelo menos metade das calorias referentes a uma porção do respectivo grupo alimentar. A soma da pontuação de cada componente gerou o escore final do IASad, a partir do qual a dieta dos participantes foi classificada em três categorias: de boa qualidade (IASad $>100$ pontos), precisando melhorar a qualidade (IASad entre 71 e 100 pontos) e de má qualidade (IASad $<71$ pontos) ${ }^{7}$.

A normalidade das variáveis em estudo foi avaliada através do teste de Kolmogorof-Smirnoff. Foram utilizados o teste $t$ de Student para amostras independentes, o teste de Mann-Whitney e o teste do Qui-quadrado, conforme indicado na comparação das características clínicas e dietéticas dos grupos (grupo E e grupo-controle). O teste Qui-quadrado de partição foi utilizado para a comparação do número de participantes do grupo $\mathrm{E}$ com os do grupo-controle, de acordo com as categorias do IASad, após detectada diferença significativa entre as categorias. A Análise de Variância (Anova one-way) foi utilizada para comparação da qualidade da dieta (pontuação final do IASad) entre os grupos, de acordo com o tem- po de acompanhamento no programa de reeducação alimentar (categorizado em tercis). O teste $t$ de Student para uma amostra foi utilizado na comparação do número de porções dos grupos alimentares da dieta dos indivíduos do grupo $\mathrm{E}$ e do grupo-controle em relação ao preconizado pelo Guia Alimentar para a População Brasileira.

Os resultados foram expressos como média \pm desvio-padrão, mediana e amplitude interquartil ( $\left.\mathrm{P}^{25}-\mathrm{P} 75\right)$ ou percentual de participantes com a característica analisada. Foram considerados estatisticamente significativos valores de $p<0,05$ (bicaudal). As análises foram realizadas no software SPSS 16.0 (SPSS Inc., Chicago, IL).

\section{RE S U LT A D OS}

Foram estudados 100 indivíduos com idade Média (M) de 40,7, Desvio-Padrão - DP=12,4 anos e IMC médio de $27,4, \mathrm{DP}=4,7 \mathrm{~kg} / \mathrm{m}^{2}$. A maioria dos participantes era do sexo feminino $(73 \%)$ e não fumante (93\%). Consumo de bebida alcoólica foi relatado por $31 \%$ dos participantes. Menos da metade (43\%) dos participantes foi classificada como sedentária. Possuíam o terceiro grau completo $67 \%$ dos participantes.

Tabela 1. Descrição, valor energético referente a uma porção e critérios para pontuação máxima e mínima dos componentes do IASad. Porto Alegre (RS), 2009.

\begin{tabular}{lccc}
\hline \multirow{2}{*}{ Itens } & $\begin{array}{c}\text { Valor } \\
\text { energético/porção }\end{array}$ & $\begin{array}{c}\text { Critério para pontuação máxima* } \\
\text { (10 pontos) }\end{array}$ & $\begin{array}{c}\text { Critério para pontuação mínima* } \\
\text { (0 pontos) }\end{array}$ \\
\cline { 2 - 4 } & \multicolumn{3}{c}{ Porções (n) } \\
\hline Cereais, pães, raízes e tubérculos & 150 & 6 & 0 \\
Hortaliças & 15 & 3 & 0 \\
Frutas & 70 & 3 & 0 \\
Leguminosas e oleaginosas & 55 & 1 & 0 \\
Carnes e ovos & 190 & 1 & 0 \\
Leite e derivados & 120 & 3 & 0 \\
Óleos e gorduras & 73 & 1 & 0 \\
Açúcares e doces & 110 & 1 & $\geq 45$ \\
Gordura total (\% do VCT) & - & $\leq 30$ & $\geq 15$ \\
Gordura saturada (\% do VCT) & - & $<10$ & $<6$ \\
Colesterol (mg) & - & $\leq 300$ & $<6$ \\
Variedade (itens diferentes/dia) & - & $>12$ & 0 \\
\hline
\end{tabular}

"Os critérios para pontuação máxima são referentes ao número de porções preconizado pelo Guia Alimentar para a população brasileira para uma dieta de 2 mil calorias.

VCT: valor calórico total. 
Na Tabela 2 estão descritas as características gerais e os dados antropométricos dos participantes do estudo, de acordo com o grupo ao qual pertenciam. As únicas diferenças observadas foram em relação ao sexo e ao grau de escolaridade dos participantes, sendo observada maior proporção de homens no grupo-controle em comparação ao grupo $\mathrm{E}$, bem como menor proporção de participantes com terceiro grau completo no primeiro grupo. Os grupos não diferiram quanto a idade, tabagismo, consumo de bebida alcoólica e percentual de participantes sedentários. Em relação aos indicadores antropométricos, não foi observada diferença entre os grupos quanto a peso, estatura, IMC e circunferência da cintura.

A composição nutricional da dieta dos participantes do programa de reeducação alimentar está apresentada na Tabela 3. O grupo-controle apresentou menor consumo de calorias, de gordura total e frações e de colesterol, assim como maior consumo de carboidrato, proteína e fibras totais.

\section{Qualidade da dieta de acordo com o IAsad}

Os componentes do IAS referentes aos grupos alimentares foram convertidos em número de porções/1 mil calorias/dia, a fim de garantir o ajuste para o valor calórico total da dieta, o qual diferiu entre os grupos. Quando estes foram comparados quanto à qualidade da dieta, foi observada maior pontuação para o IASad no grupo $\mathrm{E}$ do que no grupo NE M=97,0, DP 6,2 cfm= 84,2 $D P=15,2 ; p<0,001)$. Entretanto, a pontuação média do IASad dos dois grupos classifica a dieta de ambos como precisando melhorar a qualidade (71<IASad $<100$ pontos).

Na Tabela 4 está apresentada a pontuação para cada componente do IASad dos participantes, de acordo com a exposição a programa de reeducação alimentar. O grupo-controle apresentou maior pontuação para o grupo das frutas, hortaliças, leite e derivados, gordura total, colesterol e variedade. Já a pontuação para o grupo dos óleos e gorduras e dos açúcares e doces foi

Tabela 2. Características gerais e dados antropométricos dos participantes, de acordo com a exposição a programa de reeducação alimentar. Porto Alegre (RS), 2009.

\begin{tabular}{|c|c|c|c|c|c|}
\hline Itens & \multicolumn{2}{|c|}{ Grupo E $(n=54)$} & \multicolumn{2}{|c|}{ Grupo-Controle $(n=46)$} & $p$ \\
\hline \multicolumn{6}{|l|}{ Características gerais } \\
\hline Idade (anos) & 41,1 & 12,2 & 40,2 & 12,9 & $0,560^{\prime}$ \\
\hline Sexo masculino (\%) & \multicolumn{2}{|c|}{16,7} & \multicolumn{2}{|c|}{39,1} & $0,014^{2}$ \\
\hline Escolaridade (superior completo) (\%) & \multicolumn{2}{|c|}{81,5} & \multicolumn{2}{|c|}{34,3} & $0,001^{2}$ \\
\hline Tabagismo ativo (\%) & \multicolumn{2}{|c|}{3,7} & \multicolumn{2}{|c|}{10,9} & $0,162^{2}$ \\
\hline Consumo de bebida alcoólica (\%) & \multicolumn{2}{|c|}{29,6} & \multicolumn{2}{|c|}{32,6} & $0,748^{2}$ \\
\hline Sedentarismo (\%) & \multicolumn{2}{|c|}{35,2} & \multicolumn{2}{|c|}{52,2} & $0,087^{2}$ \\
\hline \multicolumn{6}{|l|}{ Características antropométricas } \\
\hline Peso (kg) & 76,9 & 15,6 & 74,2 & 12,6 & $0,352^{1}$ \\
\hline Estatura $(\mathrm{cm})$ & 165,0 & 8,0 & 167,3 & 9,9 & $0,203^{1}$ \\
\hline Índice de massa corporal ( $\left.\mathrm{kg} / \mathrm{m}^{2}\right)$ & 28,1 & 4,8 & 26,6 & 4,4 & $0,090^{\prime}$ \\
\hline Sobrepeso e obesidade (\%) & \multicolumn{2}{|c|}{59,7} & \multicolumn{2}{|c|}{40,3} & $0,103^{2}$ \\
\hline \multicolumn{6}{|l|}{ Circunferência da cintura (cm) } \\
\hline Homens & 102,1 & 10,7 & 95,2 & 8,2 & $0,072^{\prime}$ \\
\hline Mulheres & 91,7 & 13,4 & 87,7 & 13,0 & $0,211^{1}$ \\
\hline
\end{tabular}

1 Teste $t$ de Student - dados apresentados em média e desvio-padrão; ${ }^{2}$ Teste Qui-quadrado - dados apresentados em \% de participantes com a característica analisada; $p<0,05$ estatisticamente significativo. 
maior nos participantes do grupo-controle, não sendo observada diferença entre os grupos em relação à pontuação obtida para o grupo dos cereais, leguminosas e carnes.

Em relação à qualidade da dieta, nenhum participante do grupo E apresentou-a de má quali- dade, enquanto $70,4 \%$ tiveram-na classificada como precisando melhorar a qualidade e $29,6 \%$ apresentaram dieta de boa qualidade. Já no grupo-controle, $10,9 \%$ dos participantes apresentaram dieta de má qualidade, $71,4 \%$ precisavam melhorar a qualidade e 17,4\% apresen-

Tabela 3. Características gerais da dieta dos participantes, de acordo com a exposição a programa de reeducação alimentar. Porto Alegre (RS), 2009.

\begin{tabular}{|c|c|c|c|c|c|}
\hline \multirow{2}{*}{$\frac{\text { Dieta }}{\text { Calorias (kcal/dia) }}$} & \multicolumn{2}{|c|}{ Grupo $E(n=54)$} & \multicolumn{2}{|c|}{ Grupo-Controle $(n=46)$} & \multirow{2}{*}{$\frac{p}{<0,001^{1}}$} \\
\hline & 1925,8 & 343,5 & 2463,2 & 642,1 & \\
\hline Calorias/kg de peso/dia & 28,8 & 24,5 & 34,1 & 10,5 & $<0,001^{1}$ \\
\hline Proteína (\% do VCT) & 19,7 & 4,9 & 17,1 & 6,2 & $0,019^{1}$ \\
\hline Carboidrato (\% do VCT) & 52,4 & 7,6 & 44,9 & 10,0 & $<0,001^{1}$ \\
\hline Lipídeos (\% do VCT) & 28,0 & 6,7 & 38,0 & 9,9 & $<0,001^{1}$ \\
\hline AG Saturado (\% VCT) & 6,2 & 2,2 & 8,2 & 2,8 & $<0,001^{1}$ \\
\hline AG Monoinsaturado (\% VCT) & 5,7 & $(3,6-7,1)$ & 7,6 & $(6,0-9,5)$ & $<0,001^{2}$ \\
\hline AG Poli-insaturado (\% VCT) & 8,5 & $(3,6-11,3)$ & 12,6 & $(8,4-17,1)$ & $<0,001^{2}$ \\
\hline Colesterol (mg/dia) & 202,2 & 89,9 & 306,1 & 164,2 & $<0,001^{1}$ \\
\hline Fibras totais (g/dia) & 17,2 & 6,4 & 9,7 & 4,4 & $<0,001^{1}$ \\
\hline
\end{tabular}

${ }^{1}$ Teste $t$ de Student - dados apresentados em média e desvio-padrão; ${ }^{2}$ Teste de Mann-Whitney - dados apresentados em mediana e amplitude interquartil; $p<0,05$ estatisticamente significativo.

VCT: valor calórico total; AG: ácidos graxos.

Tabela 4. Pontuação dos componentes do IASad e classificação da qualidade da dieta dos participantes, de acordo com a exposição a programa de reeducação alimentar. Porto Alegre (RS), 2009.

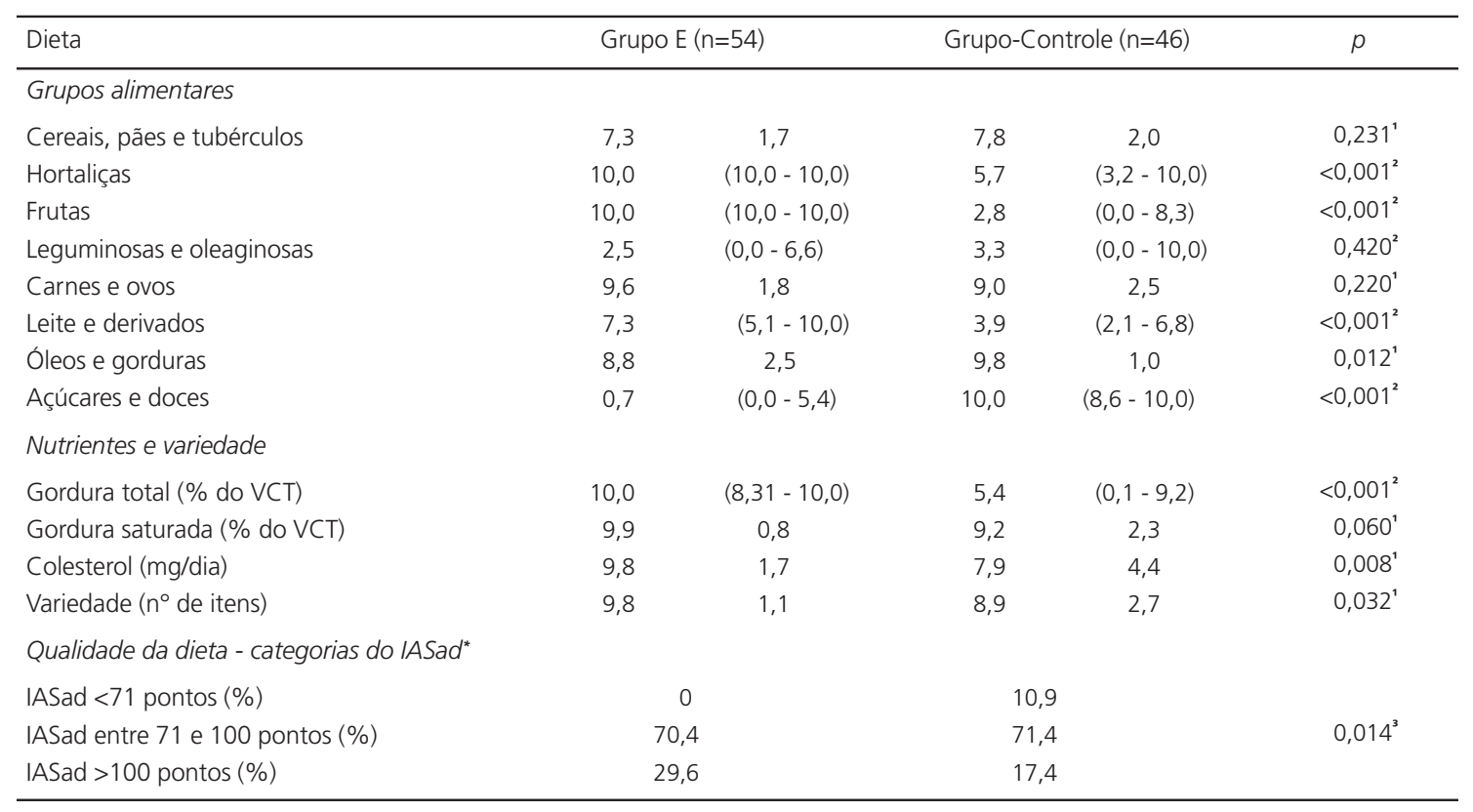

${ }^{1}$ Teste $t$ de Student - dados apresentados em média e desvio-padrão; ${ }^{2}$ Teste de Mann-Whitney - dados apresentados em mediana e amplitude interquartil; $p<0,05$ estatisticamente significativo; ${ }^{3}$ Teste Qui-quadrado de partição - dados apresentados em percentual de participantes com a característica analisada. Diferença significativa entre casos e controles nas categorias IASad $<71$ pontos e IASad >100 pontos.

VCT: valor calórico total. 
taram dieta de boa qualidade (Tabela 4). Diferença estatisticamente significativa entre os grupos foi observada na proporção de indivíduos que apresentaram dieta de má qualidade (maior no grupo-controle) e dieta de boa qualidade (maior no grupo E).

O consumo médio de porções de cada grupo alimentar, relatado pelos participantes expostos ao Programa de Reeducação Alimentar e pelos participantes do grupo-controle, foi comparado ao número de porções recomendado pelo Guia Alimentar para a população brasileira (número de porções/1 mil calorias/dia), conforme resultados apresentados na Tabela 5. Tanto o grupo E como o grupo-controle apresentou um consumo médio de porções do grupo dos cereais, leguminosas e laticínios estatisticamente inferior àquele preconizado pelo Guia Alimentar, enquanto o consumo de porções do grupo das carnes, óleos e gorduras foi significativamente maior do que a recomendação. O grupo E apresentou maior consumo de frutas e hortaliças e menor consumo de açúcares e doces em comparação ao recomendado. Já no grupo-controle, o consumo de frutas foi inferior à recomendação, e o de açúcares e doces, superior, ao passo que o consumo de hortaliças não diferiu estatisticamente do número de porções preconizados pelo Guia Alimentar.

Na Tabela 5 também estão apresentados o número e o percentual de participantes, de acordo com o grupo em estudo, que atendeu as recomentações preconizadas pelo Guia Alimentar. Observa-se que o grupo E apresentou consumo igual ou superior ao preconizado pelo Guia Alimentar para os cereais, frutas, hortaliças, leguminosas, carnes e laticínios, bem como consumo igual ou inferior ao preconizado pelo Guia Alimentar para doces e óleos, assim demonstrando ter atendido às recomendações. Por outro lado, o grupo-controle apresentou maior proporção de participantes com consumo de doces e óleos superior ao preconizado. Tais dados corroboram a informação apresentada no parágrafo anterior: indivíduos submetidos a programa de reeducação alimentar apresentaram melhor qualidade da dieta do que aqueles desprovidos de orientação nutricional prévia.

Tendo em vista a ampla variação no tempo de acompanhamento dos indivíduos submetidos ao programa de reeducação alimentar, uma análise foi conduzida a fim de comparar a qualidade da dieta dos mesmos, de acordo com o tempo de acompanhamento no programa. Para isso, os indivíduos foram divididos em tercis, pelo tempo de acompanhamento [tercil 1: menos de 8 meses ( 17 indivíduos); tercil 2: de 8 a 61 meses (19 indivíduos); tercil 3: mais de 61 meses (18 indivíduos)]. A pontuação final do IASad não diferiu entre os tercis, sendo igual a $97,74, \mathrm{DP}=5,93$ pontos no tercil 1; $95,70, \mathrm{DP}=6,88$ pontos no tercil 2; e $97,64, \mathrm{DP}=5,82$ pontos no tercil $3(p=0,540)$.

Tabela 5. Comparação entre o número de porções dos grupos alimentares do IASd/1 mil calorias consumidas e a recomendação do Guia Alimentar, de acordo com a exposição dos participantes a programa de reeducação alimentar. Porto Alegre (RS), 2009.

\begin{tabular}{|c|c|c|c|c|c|c|c|c|c|c|c|c|}
\hline \multirow[t]{3}{*}{ Grupos alimentares } & $\begin{array}{l}\text { Pirâmide } \\
\text { alimentar }\end{array}$ & $\begin{array}{l}\text { Gru } \\
(n=\end{array}$ & & \multirow[t]{3}{*}{$p^{1}$} & $\begin{array}{r}\text { Grup } \\
(n\end{array}$ & $\begin{array}{l}\text { ontrole } \\
46)\end{array}$ & \multirow[t]{3}{*}{$p^{1}$} & \multicolumn{2}{|c|}{$\begin{array}{l}\text { Grupo E } \\
(n=54)\end{array}$} & \multicolumn{2}{|c|}{$\begin{array}{c}\text { Grupo-controle } \\
(n=46)\end{array}$} & \multirow{2}{*}{$\frac{p^{1}}{\text { endaçãc }}$} \\
\hline & \multicolumn{3}{|c|}{ Porção $(n)^{3}$} & & \multicolumn{2}{|c|}{ Porção $(n)^{3}$} & & \multicolumn{4}{|c|}{ Consumo de acordo com a recomendação } & \\
\hline & & $\mathrm{M}$ & DP & & M & DP & & $n$ & $\%$ & $\mathrm{n}$ & $\%$ & \\
\hline Cereais, pães, tubérculos & 3,0 & 2,2 & 0,6 & $<0,001$ & 2,5 & 0,8 & $<0,001$ & 6 & 11,1 & 11 & 23,9 & 0,112 \\
\hline Hortaliças & 1,5 & 3,5 & 1,9 & $<0,001$ & 1,4 & 1,4 & 0,595 & 42 & 77,8 & 16 & 34,8 & $<0,001$ \\
\hline Frutas & 1,5 & 2,6 & 1,1 & $<0,001$ & 0,6 & 0,6 & $<0,001$ & 44 & 81,5 & 7 & 15,2 & $<0,001$ \\
\hline Leguminosas e oleaginosas & 0,5 & 0,2 & 0,3 & $<0,001$ & 0,3 & 0,5 & 0,018 & 7 & 13,0 & 12 & 26,1 & 0,126 \\
\hline Carnes e ovos & 0,5 & 0,9 & 0,3 & $<0,001$ & 1,1 & 0,7 & $<0,001$ & 48 & 88,9 & 37 & 80,4 & 0,272 \\
\hline Leite e derivados & 1,5 & 1,1 & 0,5 & $<0,001$ & 0,7 & 0,5 & $<0,001$ & 14 & 25,9 & 4 & 8,7 & 0,036 \\
\hline Óleos e gorduras & 0,5 & 1,2 & 0,8 & $<0,001$ & 2,2 & 1,3 & $<0,001$ & 47 & 87,0 & 12 & 26,1 & $<0,001$ \\
\hline Açúcares e doces & 0,5 & 0,2 & 0,3 & $<0,001$ & 1,0 & 0,9 & $<0,001$ & 13 & 24,1 & 3 & 6,5 & 0,027 \\
\hline
\end{tabular}

${ }^{1}$ Teste $t$ de Student para uma amostra; ${ }^{2}$ Teste Qui-quadrado; ${ }^{3}$ número de porções/1 mil calorias/dia; $p<0,05$ estatisticamente significativo. 


\section{I S C U S S Ã O}

O presente estudo comparou a qualidade da dieta de indivíduos expostos a um programa de reeducação alimentar, por um período mediano de 18 meses (o qual variou entre 2 e 169 meses) - grupo E, com a dieta de indivíduos sem orientação nutricional prévia nos últimos 6 meses - grupo-controle. Em ambos os grupos, a maior parte dos participantes foi classificada como precisando melhorar a qualidade da dieta. Entretanto, maior proporção de indivíduos com dieta de boa qualidade foi observada no grupo $E$, enquanto o grupo-controle apresentou uma proporção significativamente maior de indivíduos com dieta de má qualidade. O tempo de acompanhamento no programa de reeducação alimentar não influenciou a qualidade da dieta dos participantes do grupo E, o qual apresentou maior pontuação para vegetais, frutas, laticínios, colesterol e variedade da dieta, bem como menor pontuação para óleos e açúcares, em comparação ao grupo-controle. Estes achados apontam a reeducação alimentar como importante ferramenta na promoção de uma alimentação saudável.

A literatura científica é escassa no que se refere ao efeito da reeducação alimentar na qualidade da dieta, especialmente quando se trata de estudos que utilizam índices dietéticos. Contudo, melhora na qualidade da dieta de indivíduos, por meio da educação nutricional, é demonstrada de forma indireta por estudos disponíveis na literatura ${ }^{21,22}$. Ensaio clínico randomizado, conduzido com 80 famílias do município de São Paulo, demonstrou aumento no consumo de frutas e vegetais de famílias submetidas a encontros semanais de educação nutricional, quando comparadas às famílias do grupo-controle, as quais não receberam nenhuma intervenção educacional por três semanas ${ }^{21}$. Outro estudo avaliou o efeito de dois programas de educação nutricional sobre o risco cardiovascular em pacientes hipertensos e com excesso de peso, tendo sido demonstrada redução no consumo de calorias após 40 semanas de acompanhamento 22 .
Outros trabalhos nacionais que avaliaram a qualidade da dieta de diferentes grupos, por meio de índices dietéticos variados, também demonstraram que a maior parte dos indivíduos necessita melhorar a qualidade da dieta. Estudo realizado com moradores da cidade de Botucatu (São Paulo) demonstrou necessidade de melhoria na qualidade da dieta de $74 \%$ dos indivíduos. Nesse estudo, que utilizou o índice de qualidade da dieta, $12 \%$ dos participantes tiveram suas dietas classificadas como sendo de boa qualidade, enquanto $14 \%$ deles apresentaram dietas inadequadas ${ }^{11}$. Em outro estudo transversal nacional de base populacional, envolvendo 1840 indivíduos de ambos os sexos, $74 \%$ das dietas foram classificadas como precisando de melhorias ${ }^{12}$. Ainda, o estudo conduzido por Mota et al ${ }^{7}$., que validou e adaptou o IAS à população brasileira, demonstrou necessidade de melhorar a qualidade da dieta em $71 \%$ dos 502 paulistanos estudados.

A qualidade da dieta de 67 pacientes com diabetes melito (DM) tipo 2, avaliada através do IAS americano, foi classificada como precisando de melhorias em $52,2 \%$ dos pacientes, enquanto $40,3 \%$ apresentaram dieta de boa qualidade e os demais apresentaram dieta de má qualidade ${ }^{10}$. A maior proporção de indivíduos com dieta de boa qualidade, nesse estudo, possivelmente pode ser justificada pelo fato de os participantes apresentarem diabetes, condição clínica que geralmente leva à modificação dos hábitos alimentares, por ser a dieta um dos pilares do tratamento ${ }^{23}$. De fato, em estudo transversal envolvendo mais de 8 mil adultos americanos, a qualidade da dieta foi associada inversamente com a glicemia de jejum e a hemoglobina glicada, marcadores bioquímicos de controle glicêmico em pacientes diabéticos ${ }^{24}$.

Em relação aos componentes do IASad, os participantes expostos a programa de reeducação alimentar apresentaram maior pontuação para o grupo das frutas, vegetais, laticínios, gordura total, colesterol e variedade, bem como menor pontuação para o grupo dos açúcares e óleos. 
Entretanto, apesar de apresentarem melhor qualidade da dieta em comparação ao grupo-controle, os participantes do grupo exposto ao programa de reeducação alimentar relataram consumo médio de porções do grupo das leguminosas e do leite e derivados estatisticamente inferior à recomendação. Ademais, o consumo de alimentos do grupo das carnes e do grupo dos óleos e gorduras foi superior ao número de porções preconizado pelo Guia Alimentar para a população brasileira.

Discrepâncias em relação às recomendações nutricionais também foram encontradas por outros pesquisadores. Santos e colaboradores demonstraram consumo de vegetais, frutas e laticínios inferior à recomendação, entre pacientes com diabetes melito tipo $2^{10}$. Consumo de frutas e vegetais abaixo do preconizado também foi observado em estudo conduzido por Fisberg e colaboradores, no qual $60 \%$ dos participantes apresentaram consumo de colesterol acima do recomendado ${ }^{11}$. Baixo consumo de laticínios, frutas e verduras também foi descrito em estudo nacional de base populacional, no qual respectivamente $73 \%$ e $81 \%$ dos participantes apresentaram pontuação máxima para o consumo de carnes e de colesterol ${ }^{12}$.

A melhor qualidade da dieta de indivíduos expostos ao programa de reeducação alimentar, em comparação àqueles sem orientação nutricional prévia, observada no presente estudo, pode ser atribuída ao fato de os indivíduos do primeiro grupo terem sido orientados e acompanhados para a mudança e melhora dos hábitos alimentares, através da educação nutricional. Isso porque a educação nutricional possibilita o desenvolvimento de estratégias que modificam o padrão alimentar inadequado, bem como crenças, práticas e valores relacionados à alimentação ${ }^{25}$.

Os grupos não diferiram quanto ao estado nutricional, avaliado através do IMC e da circunferência da cintura, o que poderia confundir os resultados. A diferença no grau de escolaridade e no sexo, observada entre os grupos, poderia ser um determinante da qualidade da dieta. De fato, Morimoto et al. ${ }^{12}$ demonstraram variação significativa na qualidade da dieta de moradores da região metropolitana de São Paulo, de acordo com as características demográficas de sexo, grau de escolaridade e faixa etária. Entretanto, o tamanho amostral do presente estudo dificulta a análise dos dados, estratificada por sexo e grau de escolaridade, devendo essas variáveis ser interpretadas como potenciais confundidores dos resultados do presente estudo. Isso porque a maior proporção de mulheres e de indivíduos com escolaridade mais elevada no grupo E poderia, em parte, justificar a melhor qualidade da dieta.

O presente estudo apresenta outras limitações potenciais, que devem ser consideradas a fim de garantir a correta interpretação dos resultados. A amostra de conveniência pode não ser representativa (o que inviabiliza a generalização dos resultados), além de estar associada a um potencial viés de seleção, vinculado ao fato de que os indivíduos que aceitaram ser voluntários no estudo podem apresentar hábitos de vida distintos dos daqueles que não demonstraram interesse em participar. Além disso, a maior motivação à adoção de uma dieta de melhor qualidade entre os indivíduos do grupo $E$, que procuraram se inserir em grupos direcionados para tal, também deve ser considerada na interpretação dos resultados. A utilização do R24h não é apontada pela literatura como a melhor alternativa para avaliação do consumo alimentar, já que - entre outras limitações - depende da memória do entrevistado. Contudo, no presente estudo foram aplicados dois R24h, o que contribui para a obtenção de informações mais precisas acerca do consumo alimentar ${ }^{26}$.

\section{O N CLUSÃ O}

A qualidade da dieta de indivíduos expostos a um programa de reeducação alimentar, por um período mediano de 18 meses, foi superior à de indivíduos sem orientação nutricional prévia nos últimos 6 meses. Os resultados reforçam a importância da educação nutricional para a modificação dos hábitos alimentares e a consequente 
melhora da qualidade da dieta das populações. O consumo de laticínios e leguminosas abaixo da recomendação, e de carnes e óleos acima da recomendação, entre os indivíduos expostos a programa de reeducação alimentar, alerta para a necessidade da educação nutricional continuada. A influência da qualidade da dieta, promovida por programas de reeducação alimentar, sobre a qualidade de vida e os desfechos de saúde/doença das populações, requer a realização de novos estudos. É preciso ainda estudar melhor a influência do sexo e da escolaridade sobre a qualidade da dieta dos indivíduos submetidos a programas de reeducação alimentar.

\section{COLABORADORES}

F. FELIPPE elaborou o projeto de pesquisa, coletou e tabulou os dados e redigiu o artigo. L. BALESTRIN coletou e tabulou os dados. F.M. SILVA coorientou o trabalho, supervisionou a logística do estudo, analisou os dados e redigiu o artigo. A.P. SCHNEIDER orientou o trabalho e a supervisão da logística do estudo, redigiu o artigo.

\section{REFER Ê N CIAS}

1. Kant AK. Indexes of overall diet quality: a review. J Am Diet Assoc. 1996; 96:785-91. doi: 10.1016/S0 002-8223(96)00217-9.

2. Cervato AM, Vieira VI. Índices dietéticos na avaliação da qualidade global da dieta. Rev Nutr. 2003; 16(3):347-55. doi: 10.1590/S1415-5273 2003000300012

3. Willett WC. Nutritional epidemiology. $2^{\text {nd }}$ ed. New York: Oxford University Press; 1998.

4. Kennedy ET, Ohls J, Carlson S, Fleming K. The Healthy eating index: design and applications. J Am Diet Assoc. 1995; 95(10):103-8. doi: 10.1016/S 0002-8223(96)00217-9.

5. United States Departament of Agriculture. Center for Nutrition Policy and Promotion. Healthy Eating Index-2005: development and evaluation technical report [Internet]. Washington: USDA; 2005 [cited 2009 Mar 5]. Available from: <www.cnpp.usda. gov/HealthyEatinglndex.htm>.

6. Dietary guidelines for Americans [Internet]. Washington; 2005. [cited 2009 Mar 5]. Available from: <www.health.gov/DietaryGuidelines/dga 2005/document/default.htm>.

7. Mota JF, Rinaldi A, Pereira AF. Adaptação do índice de alimentação saudável ao guia alimentar da população brasileira Rev Nutr. 2008; 21(5):545-52. doi: 10.1590/S1415-52732008000500007.

8. Brasil. Ministério da Saúde. Guia alimentar para a população brasileira: promovendo a alimentação saudável [Internet]. Brasília; 2006 [acesso 2009 jun 10]. Disponível em: <http://dtr2004.saude.gov.br/ nutricao/guia_conheca.php>.

9. Philippi ST, Latterza AR, Cruz ATR, Ribeiro LC. Pirâmide alimentar adaptada: guia para escolha dos alimentos. Rev Nutr. 1999; 12(1):65-80. doi: 10.15 90/\$1415-52731999000100006.

10. Santos CR, Gouveia LA, Portella E, Avila SS. Índice de alimentação saudável: avaliação do consumo alimentar de diabéticos tipo 2. Nutrire: Rev Soc Bras Aliment Nutr. 2009; 34(1):115-29.

11. Fisberg RM, Slater B, Barros RR, Lima FD, Cesar CLG, Carandina $L$, et al. Índice de Qualidade da Dieta: avaliação da adaptação e aplicabilidade. Rev Nutr. 2004; 17(3):301-18. doi: 10.1590/S1415-5 2732004000300003.

12. Morimoto JM, Latorre MRDO, César CLG, Carandina L, Barros MBA, Goldbaum M, et al. Fatores associados à qualidade da dieta de adultos residentes na região metropolitana de São Paulo, Brasil, 2002. Cad Saúde Pública. 2008; 24(1): 169-78. doi: 10.1590/S0102-311X19950003000 15.

13. Drewnowski A, Hendersont SA, Shore AB, Fischler $C$, Preziosi P, Hercberg S. Diet quality and dietary diversity in France: implications for the French paradox. J Am Diet Assoc. 1996; 7(7):663-9. doi: 10.1016/S0002-8223(96)00217-9.

14. Drewnowski A, Driscoll A, Rolls BJ. The dietary variety score: assessing diet quality in healthy young and older adults. J Am Diet Assoc. 1997; 97(3): 266-71. doi: 10.1016/S0002-8223(96)00217-9.

15. Ramalho RA, Saunders C. O papel da educação nutricional no combate às carências nutricionais. Rev Nutr. 2000; 13(1):11-16. doi: 10.1590/S1415-5 2732000000100002.

16. Santos LAS. Educação alimentar e nutricional no contexto da promoção de práticas alimentares saudáveis. Rev Nutr. 2005; 18(5):681-92. doi: 10.15 90/S1415-52732005000500011.

17. Souza AC, Santos I, Costa L, Oliveira D. A educação em saúde com grupos na comunidade: uma estratégia facilitadora da promoção da saúde. Rev Gaúcha Enfermagem. 2005; 26(2):147-53. doi: 10.1590/S1983-14472010000200001. 
18. World Health Organization. Diet, nutrition and the prevention of chronic diseases. Geneva: WHO: 2003.

19. World Health Organization. Obesity: preventing and managing the global epidemic. Geneva: WHO; 1998. Report of a WHO consultation on obesity.

20. Santana RI. Avanutri: software de avaliação nutricional, versão 4.0. Rio de Janeiro; 2009.

21. Jaime PC, Machado FMS, Westphal MF, Monteiro CA. Nutritional education and fruit and vegetable intake: a randomized community trial. Rev Saúde Pública. 2007; 41(1). doi: 10.1590/S0034-891020 09000900001.

22. Alvarez TS, Zanella MT. Impacto de dois programas de educação nutricional sobre o risco cardiovascular em pacientes hipertensos e com excesso de peso. Rev Nutr. 2009; 22(1):71-9. doi: 10.1590/\$1415-5 2732009000100007.

23. American Diabetes Association. Nutrition recommendations and interventions for diabetes: a position statement of the American Diabetes Association. Diabetes Care. 2008; 31(1):61-78. doi: 10.2337/dc10-S001.

24. Kant AK, Graubard BI. A comparison of three dietary pattern indexes for predicting biomarkers of diet and disease. J Am Coll Nutr. 2005; 24(4): 294-303.

25. Assis MA, Nahas M. Aspectos motivacionais em programas de mudança de comportamento alimentar. Rev Nutr. 1999; 12(1):33-41. doi: 10.1590/S 1415-52731999000100002.

26. Fisberg RM, Marchioni DML, Colucci ALA. Avaliação do consumo alimentar e da ingestão de nutrientes na prática clínica. Arq Bras Endocrinol Metab. 2009; 53(5):617-24. doi: 10.1590/S0004-27302004000 200014.

Recebido em: 29/6/2010

Versão final reapresentada em: 4/7/2011 Aprovado em: 25/8/2011 